\title{
A fishy option for human norovirus research
}

Van Dycke, J., et al. PloS Pathog 15, e1008009 (2019)

Norovirus may be the leading global cause of foodborne illness-the highly contagious virus triggers acute gastroenteritis in almost 700 million people annually and contributes to 200,000 deaths-but it's proven a tricky bug to study. It can be a challenge to cultivate in the lab relative to other viruses, explains pharmacist-turnedbiologist Joana Rocha-Pereira of KU Leuven in Belgium; until the 1990s, it was difficult to even detect in the first place. Since then, researchers have gotten a handle on its epidemiology and physical structure, but gaps remain in our basic understanding of norovirus biology, she says. What are its receptors in its host? How does it replicate and come to infect immune cells, in addition to enteric ones?

Answering those questions has been limited by a dearth of animal options in which to model human norovirus infection-particularly small animals that can be used in larger numbers and at less cost than gnotobiotic pigs or calves. Rocha-Pereira had been working with a mouse model, but one infected with a murine-specific norovirus; studying that surrogate viral form sufficed for a while but, with an eye towards moving preclinical drug candidates along the testing pipeline, she wanted to work with human norovirus itself. Then one day, she was introduced to zebrafish.

Another group at KU Leuven was using larval zebrafish to study epilepsy, but a look into the literature revealed infection models of human influenza, chikungunya, and herpes simplex - three very different viruses with very different means of infection and replication, she says. Human norovirus seemed worth a try-and it worked. Writing in the journal PloS Pathogens, Rocha-Pereira and her colleagues describe their larval zebrafish model of human norovirus infection.

There were number of practical challenges along the way to developing the model. There was the question of how to deliver the virus itself. As it can be difficult to gavage such small animals, the team decided to inject stool samples from human norovirus patients into the egg yolk of three day post-fertilization zebrafish; as norovirus

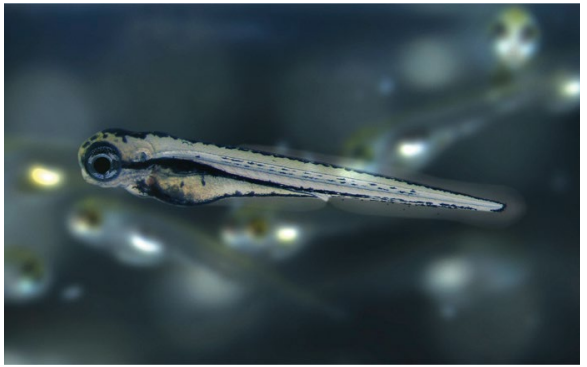

A new study reveals that human norovirus can infect and replicate within larval zebrafish. Credit: Paulo Oliveira / Alamy Stock Photo

initially targets the enteric system, delivery via the yolk, which feeds the larvae in those early developmental days, seemed the most analogous option to mimic the food-borne route by which humans become infected, Rocha-Pereira says. Given that they were working with a virus capable of infecting humans, they also had to figure out the most efficient way to inject many, many miniscule fish within the confines of a laminar flow cabinet; a microscope with a large screen helped.

Although the fish did not become visible sick, human norovirus-of several different viral genotypes-did replicate within the larvae, peaking after two days but remaining detectable for about a week. The team observed increased expression in several genes involved with the innate immune system, and the virus was also detected in other tissues. These included caudal hematopoietic tissue. A broad-spectrum antiviral treatment, meanwhile, reduced viral replication.

"I think this is a great advance that has clear translational implications," commented Christiane Wobus from the University of Michigan Medical School, who has developed a human norovirus mouse model (Wobus has collaborated with the KU Leuven group in the past but was not involved in the current zebrafish work). She cautions, however, that no model is perfect; the zebrafish overcomes some of the issues with human norovirus mouse model-such as infection with multiple viral genotypes, larger viral increases over time, ability to passage, much smaller inoculation volumes, etc-but not all. The fish model involves a non-physiological route of inoculation requiring a high titer stool sample, and there's no ability to generate a virus stock from the fish, she noted. "Given the tools available for the zebrafish model though, new basic science advances will surely follow from this discovery and that is always a win in my opinion. So I am very excited about this finding!"

Rocha-Pereira says the lab is already using the model for antiviral drug testing. "Being a vertebrate model but still very, very simple, it can be very powerful," she says. "It's something you can put inside a 96 well plate...That's almost as simple as running an in vitro experiment." But rather than cells, it's a whole organism being studied. Because it's so very small, only small amounts of new drugs need to be synthesized for preliminary testing; that can save time, money, and effort, while garnering additional biological detail at earlier time points relative to typical pipelines involving rodents, she says.

Beyond drug screening, the many tools to make any number of different transgenic zebrafish lines might also help the researchers more closely follow the virus within the transparent larvae. "If we want to look at the role of macrophages for example, there are transgenic lines that have fluorescent macrophages," she says. After infection, what those macrophages do in response can easily be observed in the fish under a microscope. "That's very powerful," she says. "It's not so easy to do with other furry animals."

The fish indeed won't answer every human norovirus question, but RochaPereira is excited to move forward with the new model. "Finally, I can work with the real thing," she says. "We are working on the human viruses that are actually making people sick-it's not some lab-adapted adapted strain people use in cell cultures."

Stomach flu research might have a fishy future.

\section{Ellen P. Neff}

Published online: 14 October 2019

https://doi.org/10.1038/s41684-019-0427-2 